\title{
Plasma Energy-Balance Metabolites Discriminate Asymptomatic Patients with Peripheral Artery Disease
}

\author{
Anna Hernández-Aguilera ${ }^{(D)},{ }^{1}$ Salvador Fernández-Arroyo, ${ }^{2}$ Noemí Cabre, ${ }^{2}$ \\ Fedra Luciano-Mateo $\left(\mathbb{D},{ }^{2}\right.$ Gerard Baiges-Gaya, ${ }^{2}$ Montserrat Fibla, ${ }^{1}$

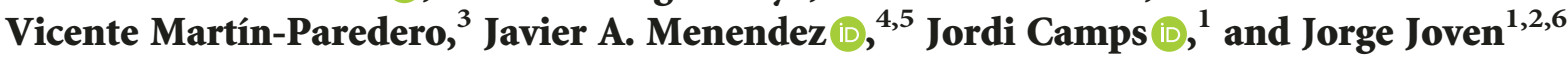 \\ ${ }^{1}$ Unitat de Recerca Biomèdica, Hospital Universitari Sant Joan, Institut d'Investigació Sanitària Pere Virgili, C. Sant Joan s/n, \\ 43201 Reus, Spain \\ ${ }^{2}$ Universitat Rovira $i$ Virgili, C. Escorxador s/n, 43003 Tarragona, Spain \\ ${ }^{3}$ Department of Vascular Surgery, Hospital Universitari Joan XXIII, C/ Dr. Mallafré Guasch 4, 43005 Tarragona, Spain \\ ${ }^{4}$ Program Against Cancer Therapeutic Resistance (ProCURE), Metabolism and Cancer Group, Catalan Institute of Oncology, \\ Girona, Spain \\ ${ }^{5}$ Girona Biomedical Research Institute (IDIBGI), Girona, Spain \\ ${ }^{6}$ The Campus of International Excellence Southern Catalonia, Tarragona, Spain
}

Correspondence should be addressed to Jordi Camps; jcamps@grupsagessa.com

Received 16 March 2018; Accepted 15 July 2018; Published 20 September 2018

Academic Editor: Adrian Doroszko

Copyright (c) 2018 Anna Hernández-Aguilera et al. This is an open access article distributed under the Creative Commons Attribution License, which permits unrestricted use, distribution, and reproduction in any medium, provided the original work is properly cited.

\begin{abstract}
Peripheral artery disease (PAD) is a common disease affecting $20-25 \%$ of population over 60 years old. Early diagnosis is difficult because symptoms only become evident in advanced stages of the disease. Inflammation, impaired metabolism, and mitochondrial dysfunction predispose to $\mathrm{PAD}$, which is normally associated with other highly prevalent and related conditions, such as diabetes, dyslipidemia, and hypertension. We have measured energy-balance-associated metabolite concentrations in the plasma of PAD patients segregated by the severity of the disease and in plasma of healthy volunteers using a quantitative and targeted metabolomic approach. We found relevant associations between several metabolites (3-hydroxybutirate, aconitate, (iso)citrate, glutamate, and serine) with markers of oxidative stress and inflammation. Metabolomic profiling also revealed that (iso)citrate and glutamate are metabolites with high ability to discriminate between healthy participants and PAD patients without symptoms. Collectively, our data suggest that metabolomics provide significant information on the pathogenesis of PAD and useful biomarkers for the diagnosis and assessment of progression.
\end{abstract}

\section{Introduction}

The prevalence of peripheral artery disease $(\mathrm{PAD})$ is now higher than $20 \%$ in population over 60 years, and affected patients have a several-fold increased risk of all-cause mortality compared to people without the disease [1]. Age, hypertension, hypercholesterolemia, diabetes, and smoking are recognized risk factors, but the disease progresses silently for decades, with the consequence that if appropriate, effective measures are applied too late, or not implemented at all, will result in atherosclerosis affecting wide portions of the arteries in the lower extremities [2]. Tissue ischemia is a common finding in PAD patients, and an increasing body of evidence supports the notion that inflammation plays an important role in the pathogenesis of this disease, linking oxidative stress, metabolic adaptation, and immunity [3-6]. Consequently, the combined action of abnormal mitochondrial function, increased production of reactive oxygen spe- 
TABLe 1: Clinical characteristics, blood count, and biochemical characteristics of the control group and PAD patients.

\begin{tabular}{|c|c|c|c|}
\hline & Control $(n=48)$ & $\operatorname{PAD}(n=201)$ & $p$ value \\
\hline BMI $\left(\mathrm{kg} / \mathrm{m}^{2}\right)$ & $24(22.5-25.3)$ & $25(22.5-28)$ & 0.021 \\
\hline Diabetes (\%) & - & 64.1 & $<0.001$ \\
\hline Hypertension (\%) & - & 69.2 & $<0.001$ \\
\hline Dyslipidemia & - & 37.9 & $<0.001$ \\
\hline Red blood cells, $\times 10^{12} / 1$ & $4.9(4.4-5.2)$ & $4.16(3.57-4.66)$ & $<0.001$ \\
\hline Hemoglobin, mmol/l & $8.94(8.32-9.43)$ & $13.30(11.50-14.90)$ & 0.001 \\
\hline Leukocytes, $\times 10^{9} / 1$ & $6.8(5.4-8.2)$ & $8.17(6.50-10.22)$ & 0.003 \\
\hline Platelets, $\times 10^{9} / 1$ & $233(205-273)$ & $253(200-329)$ & ns \\
\hline Total cholesterol, mmol/l & $4.85(4.40-5.85)$ & $3.90(3.31-4.94)$ & $<0.001$ \\
\hline HDL cholesterol, mmol/l & $1.34(1.14-1.61)$ & $0.96(0.78-1.19)$ & $<0.001$ \\
\hline LDL cholesterol, mmol/l & $2.82(2.40-3.86)$ & $2.26(1.77-2.79)$ & $<0.001$ \\
\hline Triglycerides, mmol/l & $0.90(0.70-1.38)$ & $1.99(1.40-3.08)$ & $<0.001$ \\
\hline Glucose, $\mathrm{mmol} / \mathrm{l}$ & $4.70(4.37-4.92)$ & $5.61(4.60-6.88)$ & $<0.001$ \\
\hline $\mathrm{ALT}, \mathrm{U} / \mathrm{l}$ & $20(13.5-24.9)$ & $21.5(15-34.8)$ & ns \\
\hline AST, U/l & $20(17.7-24)$ & $21(16-32)$ & ns \\
\hline
\end{tabular}

BMI: Body mass index; HDL: high-density lipoprotein; LDL: low-density lipoprotein; ALT: alanine aminotransferase; AST: aspartate aminotransferase; ns: not significant. Nonparametric variables are shown as medians, and IQR are in parentheses. Qualitative variables are expressed as percentage of total participants.

cies, impaired energy metabolism, and the subsequent inflammatory response complicates vascular remodeling, perfusion recovery, and atherosclerosis [7-10].

One of the main challenges clinicians face is early diagnosis of PAD (i.e., during the asymptomatic stages), and many biomarker candidates have been proposed with limited success [11-13]. The emerging metabolomic approaches are providing new clues in understanding atherosclerosis-related cardiovascular disease [14-20], but efforts on PAD have been scarce. Previous studies from our group suggested that serum paraoxonase-1 (PON1) and the chemokine (C-C) motif ligand 2 (CCL2) might be useful biomarkers of PAD [21, 22]: PON1 being a scavenger of excessive reactive oxygen species [23] and CCL2 an inducer of monocyte migration and differentiation into macrophages [24]. As mitochondrial dysfunction and inflammation result in disrupted metabolism $[25,26]$, we report here that metabolomic profiling of plasma may be useful for identifying patients at increased risk of PAD and that energy-balance metabolites are associated with inflammation and oxidation in these patients. Our results might suggest new biomarkers and therapeutic targets.

\section{Materials and Methods}

2.1. Participants and Study Design. We performed an observational, cross-sectional study in 201 men with clinically diagnosed PAD attending our vascular surgery department between 2010 and 2015. Patients were classified according to the Fontaine classification in nonsymptomatic stage (grade I), intermittent claudication (grade II), rest pain (grade III), and tissue damage, and necrosis (grade IV) [27]. Diagnostic criteria involved ankle-brachial index (ABI), noninvasive imaging techniques (computerized tomography scan or magnetic resonance imaging), and arteriography when indicated. The exclusion criteria were the presence of acute ischemia, signs of infection, renal failure, liver disease, cancer, or autoimmune disease. Clinical data and laboratory variables were obtained from patients' clinical records. For comparisons, we used biobanked samples $(n=48)$ from healthy, agematched men and normal ABI values, whose details have been previously described [28]. Plasma and serum samples from all participants were collected and stored at $-80^{\circ} \mathrm{C}$ until use. Procedures were approved by the Comité d'Ėtica d'Investigació Clínica of Hospital Universitari de Sant Joan (EPINOLS/1203-09/3proj6 and INFLAMET/15-04-30/4proj6), and written informed consent was obtained from all participants.

2.2. Metabolomic Analyses. To detect and quantify metabolites of energy metabolism, we used a previously described method [6]. Briefly, metabolites from plasma $(100 \mu \mathrm{l})$ were extracted using $400 \mu \mathrm{l}$ of methanol/water $(8: 2, v / v)$ and proteins were precipitated for two hours at $-20^{\circ} \mathrm{C}$. After centrifugation at $14,000 \mathrm{rpm}$ for 10 minutes at $4^{\circ} \mathrm{C}$, the supernatant was collected and dried under $\mathrm{N}_{2}$ flow. Metabolites were then derivatized with methoxyamine in pyridine (40 mg/ml) and N-methyl-N-(trimethylsilyl)-trifluoroacetamide and injected into a gas chromatograph coupled with a quadrupole time-of-flight mass spectrometer by an electron impact source. Metabolites were detected and quantified with the use of proper calibration curves.

2.3. Biochemical Analyses. The true physiological substrates for PON1 have not been yet identified. Since PON1 has lactonase and esterase activities [23], we opted to analyze the catalytic activity of PON1 using two different substrates: paraoxon (an ester) and 5-thiobutyl butyrolactone (TBBL; a synthetic lactone). Serum PON1 paraoxonase activity was determined as the rate of hydrolysis of paraoxon at $410 \mathrm{~nm}$ and $37^{\circ} \mathrm{C}$ in a $0.05 \mathrm{mM}$ glycine buffer, $\mathrm{pH} 10.5$ with $1 \mathrm{mM}$ $\mathrm{CaCl}_{2}$. Activities were expressed as $\mathrm{U} / \mathrm{l}(1 \mathrm{U}=1 \mu \mathrm{mol}$ of paraoxon hydrolyzed per minute). Serum PON1 lactonase activity was measured in an assay containing $1 \mathrm{mM} \mathrm{CaCl}_{2}$, 


\begin{tabular}{|c|c|c|c|c|}
\hline Metabolite & Control $(\mu \mathrm{M})$ & $\operatorname{PAD}(\mu \mathrm{M})$ & $\begin{array}{c}\text { PAD-to-control } \\
\text { ratio }\end{array}$ & $\rho$-value \\
\hline 3-Hydroxybutirate & $0.12(0.11-0.13)$ & $0.22(0.14-0.40)$ & 1.83 & $<0.001$ \\
\hline Aconitate & $0.47(0.39-0.63)$ & $4.42(2.45-6.26)$ & 9.40 & $<0.001$ \\
\hline$\alpha$-Ketoglutarate & $2.91(2.15-3.97)$ & $4.67(2.85-7.07)$ & 1.60 & $<0.001$ \\
\hline Alanine & $199.95(163.83-252.32)$ & $208.71(147.51-274.36)$ & 1.04 & ns \\
\hline Aspartate & $132.46(114.27-147.78)$ & $181.61(142.32-229.68)$ & 1.37 & $<0.001$ \\
\hline (Iso)citrate & $267.20(200.34-329.55)$ & $687.82(561.31-921.93)$ & 2.57 & $<0.001$ \\
\hline Fumarate & $0.31(0.23-0.41)$ & $0.25(0.19-0.39)$ & -1.24 & 0.032 \\
\hline Glucose & $4718.45(4407.71-5073.74)$ & $4878.41(4312.48-5658.30)$ & 1.03 & ns \\
\hline Glutamate & $168.57(106.17-258.28)$ & $1417.56(711.82-2669-54)$ & 8.41 & $<0.001$ \\
\hline Glutamine & $1705.99(990.44-2736.23)$ & $5073.46(2616.13-9189.28)$ & 2.97 & $<0.001$ \\
\hline Isoleucine & $48.28(40.66-55.59)$ & $61.55(52.22-73.56)$ & 1.27 & $<0.001$ \\
\hline Lactate & $399.68(348.17-429.08)$ & $358.59(305.02-440.07)$ & -1.11 & ns \\
\hline Leucine & $71.25(62.95-80.77)$ & $88.41(71.57-107.83)$ & 1.24 & $<0.001$ \\
\hline Malate & $1.43(1.15-1.77)$ & $2.51(1.90-3.88)$ & 1.76 & $<0.001$ \\
\hline Pyruvate & $9.12(6.47-13.49)$ & $13.49(3.79-23.04)$ & 1.48 & ns \\
\hline Serine & $103.49(93.41-112.86)$ & $135.86(105.32-167.17)$ & 1.31 & $<0.001$ \\
\hline Succinate & $10.52(10.25-11.09)$ & $9.40(8.44-15.07)$ & -1.12 & 0.021 \\
\hline Succinyl-CoA & $7.27(5.27-9.76)$ & $10.45(7.58-15.54)$ & 1.44 & $<0.001$ \\
\hline Valine & $92.81(82.87-104.55)$ & $105.44(83.91-135.10)$ & 1.14 & 0.004 \\
\hline
\end{tabular}

(a)

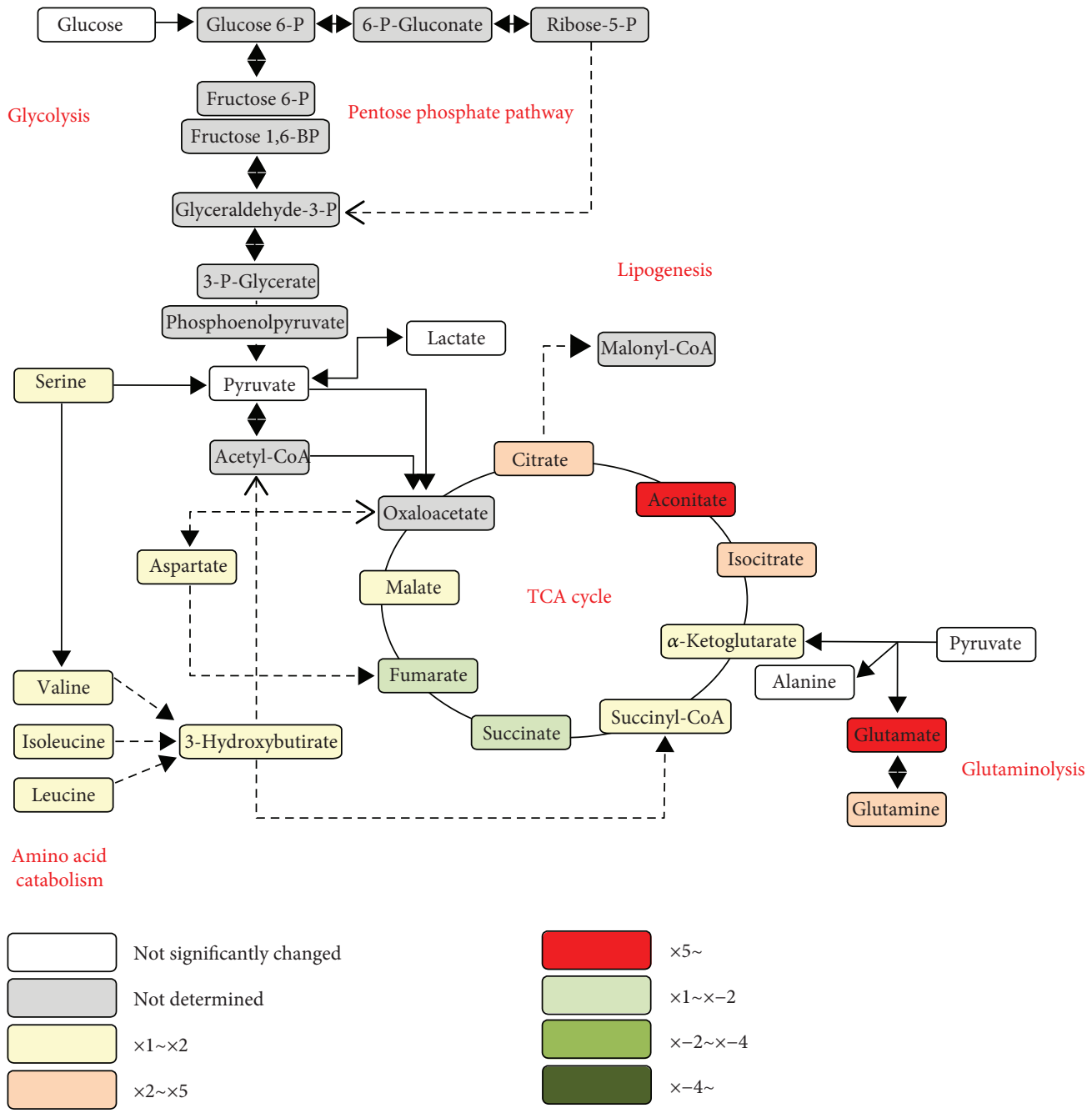

(b)

Figure 1 
TABle 2: Spearman's correlation coefficients for PON1 concentration, PON1 paraoxonase activity, PON1 lactonase activity, CCL2 concentration, and related metabolites.

\begin{tabular}{|c|c|c|c|c|c|c|c|c|}
\hline & \multicolumn{2}{|c|}{ PON1 concentration } & \multicolumn{2}{|c|}{ Paraoxonase activity } & \multicolumn{2}{|c|}{ Lactonase activity } & \multicolumn{2}{|c|}{ CCL2 concentration } \\
\hline & Spearman's rho & $p$ value & Spearman's rho & $p$ value & Spearman's rho & $p$ value & Spearman's rho & $p$ value \\
\hline 3-hydroxybutirate & -0.640 & $<0.001$ & -0.309 & $<0.001$ & -0.496 & $<0.001$ & 0.241 & 0.009 \\
\hline$\alpha$-Ketoglutarate & -0.021 & ns & -0.183 & 0.029 & -0.029 & ns & -0.045 & ns \\
\hline Aconitate & -0.641 & $<0.001$ & -0.394 & $<0.001$ & -0.552 & $<0.001$ & 0.345 & $<0.001$ \\
\hline Alanine & 0.153 & ns & 0.253 & 0.002 & 0.275 & 0.001 & -0.155 & ns \\
\hline Aspartate & -0.241 & 0.004 & -0.187 & 0.026 & -0.349 & $<0.001$ & 0.184 & 0.046 \\
\hline (Iso)citrate & -0.592 & $<0.001$ & -0.166 & 0.048 & -0.381 & $<0.001$ & 0.303 & 0.001 \\
\hline Fumarate & 0.295 & $<0.001$ & -0.053 & ns & 0.166 & 0.048 & -0.014 & ns \\
\hline Glucose & 0.011 & ns & 0.109 & ns & 0.053 & ns & -0.098 & ns \\
\hline Glutamate & -0.590 & $<0.001$ & -0.425 & $<0.001$ & -0.589 & $<0.001$ & .0171 & ns \\
\hline Glutamine & -0.263 & 0.002 & -0.059 & ns & -0.198 & 0.018 & 0.187 & 0.043 \\
\hline Isoleucine & -0.342 & $<0.001$ & -0.101 & ns & -0.299 & $<0.001$ & 0.167 & ns \\
\hline Lactate & 0.277 & 0.001 & 0.086 & ns & 0.175 & 0.036 & -0.104 & ns \\
\hline Leucine & -0.229 & 0.006 & 0.008 & ns & -0.136 & ns & 0.079 & ns \\
\hline Malate & -0.356 & $<0.001$ & -0.240 & 0.004 & -0.279 & 0.001 & 0.225 & 0.014 \\
\hline Pyruvate & 0.356 & $<0.001$ & -0.034 & ns & 0.158 & ns & -0.163 & ns \\
\hline Serine & -0.595 & $<0.001$ & -0.294 & $<0.001$ & -0.534 & $<0.001$ & 0.295 & 0.001 \\
\hline Succinate & -0.355 & $<0.001$ & -0.194 & 0.021 & -0.342 & $<0.001$ & 0.110 & ns \\
\hline Succinyl-CoA & -0.223 & 0.008 & -0.101 & ns & -0.154 & ns & 0.025 & ns \\
\hline Valine & -0.035 & ns & 0.155 & ns & 0.076 & ns & -0.009 & ns \\
\hline
\end{tabular}

PON1: Paraoxonase 1; CCL2: chemokine (C-C) motif ligand 2; ns: not significant.

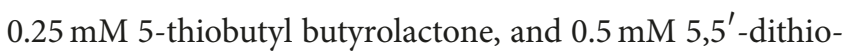
bis-2-nitrobenzoic acid (DTNB) in $0.05 \mathrm{mM}$ Tris- $\mathrm{HCl}$ buffer, $\mathrm{pH}=8.0$. The change in absorbance was monitored at $412 \mathrm{~nm}$. Activities were expressed as $\mathrm{U} / \mathrm{l}(1 \mathrm{U}=1 \mathrm{mmol}$ of TBBL hydrolyzed per minute) [29]. Serum PON1 concentrations were determined by an in-house enzyme-linked immunosorbent assay (ELISA) with a rabbit polyclonal antibody generated against the synthetic peptide CRNHQSSYQTRLNALREVQ which is sequence specific for mature PON1 [30]. CCL2 metabolites were measured by ELISA (PeproTech, London, UK). Serum lipid, liver profiles, and glucose concentrations were analyzed by standard tests in a Roche Modular Analytics P800 system. Blood count was analyzed in a Roche Sysmex XT-1800i counter (Roche Diagnostics, Basel, Switzerland).

2.4. Statistical Analysis. Differences between groups were assessed by Student's $t$-test (parametric) or Mann-Whitney $U$ test (nonparametric). Correlations between variables were assessed by Spearman's rho test. The relative magnitude of observed changes was evaluated using random forest analysis [31]. Receiver operating characteristic (ROC) curves were used to assess the diagnostic accuracy of the measured variables. This analysis represents plots of all the sensitivity/specificity pairs resulting from varying decision thresholds. Sensitivity (or true-positive rate) is the proportion of the sample correctly identified as having a specific disease. Specificity (or true-negative rate) is the proportion of the subjects correctly identified as not having a specific disease. False-positive rate is calculated as
1 - specificity. The area under the curve (AUC) and 95\% confidence interval (CI) were calculated. The AUC represents the ability of the test to correctly classify patients according to the investigated alteration. The values of AUC can range between 1 (perfect test) and 0.5 (worthless test) [32]. Statistical analyses were performed with SPSS 22.0 (IBM Corp., Chicago, IL, USA). MetaboAnalyst 3.0 (http://www.metaboanalyst.ca/) was used to generate scores/loading plots and random forest analyses.

\section{Results}

3.1. Participants' Characteristics. Clinical characteristics and biochemical variables of PAD patients and the control group are shown in Table 1. PAD patients had a mildly higher BMI than those in the control group, and they often had diabetes, hypertension, or dyslipidemia. Serum glucose and triglyceride concentrations were higher in patients, while cholesterol was lower, which is probably the result of medication.

3.2. Alterations in Energy-Balance-Associated Metabolites. The plasma concentrations of most energy metabolism intermediates were significantly higher in PAD patients than those in the control group, with the exceptions of fumarate, lactate, and succinate, that were decreased (Figure 1(a)). Glutaminolysis was disrupted in PAD, as shown by glutamate and glutamine increases. Moreover, reactions involving amino acid metabolism seemed to be inhibited in PAD, as serine, valine, isoleucine, and leucine concentrations were increased. TCA cycle was strongly disturbed since 
TABLE 3: Metabolite concentration in patients segregated according to the presence of diabetes.

\begin{tabular}{lcc}
\hline Metabolite & No & Yes \\
\hline 3-hydroxybutirate & $0.27(0.13-0.39)$ & $0.27(0.14-0.40)$ \\
Aconitate & $3.75(2.38-6.50)$ & $4.60(2.67-6.28)$ \\
$\alpha$-Ketoglutarate & 4.24 & $4.13(2.76-6.50)$ \\
Alanine & $(2.56-7.22)$ & $189.6(139.0-271.4)$ \\
Aspartate & $210.6(147.5-266.3)$ & $179.7(143.0-225.1)$ \\
(Iso)citrate & $172.5(133.3-215.8)$ & $665.6(538.0-880.5)$ \\
Fumarate & $0.26(584.42-867.3)$ & $0.24(0.18-0.37)$ \\
Glucose & $4546.0(4104.0-5115.5)$ & $(4040.8-5804.9)^{\mathrm{a}}$ \\
Glutamate & $1457.2(743.0-2912.4)$ & $1416.9(671.7-2684.2)$ \\
Glutamine & $5073.4(3054.2-7754.5)$ & $4742.5(1842.3-8881.2)$ \\
Isoleucine & $57.14(47.71-63.99)$ & $63.68(52.83-75.20)^{\mathrm{a}}$ \\
Lactate & $367.2(283.9-423.6)$ & $341.0(297.1-441.6)$ \\
Leucine & $85.07(70.62-94.86)$ & $86.68(69.33-109.65)$ \\
Malate & $2.38(1.85-4.31)$ & $2.45(1.88-3.56)$ \\
Pyruvate & $12.47(3.64-22.87)$ & $12.83(3.55-21.89)$ \\
Serine & $147.5(101.7-167.4)$ & $137.6(109.4-169.5)$ \\
Succinate & $9.57(8.35-15.11)$ & $9.70(8.47-15.23)$ \\
Succinyl-CoA & $10.14(7.74-15.12)$ & $10.46(7.37-17.62)$ \\
Valine & $102.0(83.0-129.4)$ & $104.1(80.7-138.9)$ \\
\hline a $<<0.01$. & &
\end{tabular}

TABLE 4: Metabolite concentrations in patients segregated according to the presence of hypertension.

\begin{tabular}{lcc}
\hline \multirow{2}{*}{ Metabolite } & No & Hypertension \\
\hline 3-hydroxybutirate & $0.21(0.14-0.38)$ & $0.35(0.14-0.42)$ \\
Aconitate & $4.59(2.54-6.65)$ & $4.13(2.60-6.60)$ \\
$\alpha$-Ketoglutarate & $4.71(2.81-6.67)$ & $3.70(2.51-7.07)$ \\
Alanine & $213.5(152.7-273.7)$ & $184.3(137.3-255.1)$ \\
Aspartate & $181.6(141.4-239.5)$ & $173.1(137.3-200.4)$ \\
(Iso)citrate & $721.8(566.6-934.0)$ & $654.2(476.5-815.9)$ \\
Fumarate & $0.27(0.19-0.40)$ & $0.22(0.17-0.30)^{\mathrm{a}}$ \\
& & 4418.1 \\
Glucose & $4955.9(4459.1-5679.9)$ & $(4134.8-5191.7)^{\mathrm{b}}$ \\
Glutamate & $1335.8(725.0-2628.5)$ & $1786.6(691.1-2891.7)$ \\
Glutamine & $5083.3(2842.0-9072.2)$ & $4742.5(1700.9-7003.8)$ \\
Isoleucine & $63.52(52.24-75.19)$ & $57.88(51.23-63.22)^{\mathrm{a}}$ \\
Lactate & $373.5(308.3-452.5)$ & $332.3(279.5-405.9)^{\mathrm{a}}$ \\
Leucine & $90.17(71.33-109.35)$ & $84.54(69.77-93.84)$ \\
Malate & $2.79(1.96-4.09)$ & $2.23(1.75-3.08)^{\mathrm{a}}$ \\
Pyruvate & $13.86(3.85-24.11)$ & $8.78(2.72-18.15)^{\mathrm{a}}$ \\
Serine & $135.3(106.1-162.1)$ & $161.0(103.6-172.0)^{\mathrm{a}}$ \\
Succinate & $9.15(8.42-15.04)$ & $11.71(8.49-15.47)$ \\
Succinyl-CoA & $11.21(7.72-16.31)$ & $9.94(7.52-14.25)$ \\
Valine & $107.3(86.6-136.8)$ & $96.6(78.8-134.8)$ \\
\hline a $p<0.05 ;{ }^{\mathrm{b}} p<0.01$. &
\end{tabular}

TABLE 5: Metabolite concentrations in patients segregated according to the presence of dyslipidemia.

\begin{tabular}{lcc}
\hline Metabolite & No & Dyslipidemia \\
\hline 3-hydroxybutirate & $0.31(0.16-0.40)$ & $0.19(0.13-0.419)$ \\
Aconitate & $4.64(2.91-6.61)$ & $4.22(2.36-6.16)$ \\
$\alpha$-Ketoglutarate & $3.99(2.44-6.55)$ & $5.05(3.05-6.90)$ \\
Alanine & $180.5(125.1-247.5)$ & $233.6(171.4-302.8)^{\mathrm{b}}$ \\
Aspartate & $168.6(134.1-205.4)$ & $191.7(148.8-253.2)^{\mathrm{a}}$ \\
(Iso)citrate & $678.6(545.4-864.5)$ & $712.3(566.1-959.1)$ \\
Fumarate & $0.23(0.18-0.36)$ & $0.27(0.19-0.40)$ \\
& 4663.9 & 4925.3 \\
Glucose & $(4172.3-5417.5)$ & $(4503.6-5684.9)^{\mathrm{a}}$ \\
Glutamate & $1556.2(679.8-2785.7)$ & $1342.5(763.7-2372.1)$ \\
Glutamine & $4742.5(2007.9-7169.0)$ & $(2219.3-10427.7)$ \\
Isoleucine & $57.90(49.81-68.46)$ & $65.02(55.35-77.95)^{\mathrm{b}}$ \\
Lactate & $334.7(274.2-435.6)$ & $393.3(323.2-457.6)^{\mathrm{b}}$ \\
Leucine & $83.96(66.70-98.71)$ & $92.19(76.10-109.92)^{\mathrm{a}}$ \\
Malate & $2.37(1.83-3.66)$ & $2.87(2.12-3.80)$ \\
Pyruvate & $9.50(3.08-21.00)$ & $13.58(6.00-25.78)$ \\
Serine & $150.0(104.9-170.3)$ & $131.9(106.0-18.8)$ \\
Succinate & $10.82(8.44-15.23)$ & $9.11(8.46-15.15)$ \\
Succinyl-CoA & $9.79(6.71-14.00)$ & $13.36(8.90-18.47)^{\mathrm{b}}$ \\
Valine & $99.3(78.6-125.5)$ & $113.3(89.6-139.4)^{\mathrm{b}}$ \\
\hline a $p<0.05 ;{ }^{\mathrm{b}} p<0.01$. & &
\end{tabular}

(iso)citrate, aconitate, $\alpha$-ketoglutarate, succinyl-CoA, and malate concentrations were increased, and fumarate and succinate concentrations were decreased in PAD (Figure 1(b)).

3.3. Relationships between Energy-Balance-Associated Metabolites, PON1 and CCL2. We observed significant inverse correlations between metabolites and PON1-related variables and direct correlations between metabolites and CCL2. The strongest correlations were observed between serum PON1 concentration and PON1 lactonase activity with 3-hydroxybutirate, aconitate, (iso)citrate, glutamate, and serine (Table 2).

3.4. Metabolites Are Linked to PAD Comorbidities, Age, and $B M I$. Univariate analyses confirmed that many metabolites were associated with diabetes, hypertension, or dyslipidemia (Tables 3-5), while multivariate analyses (principal component analyses) revealed that the combination of these metabolites was not able to separate groups regarding hypertension (Figure 2(a)) and dyslipidemia (Figure 2(b)). Random forest analysis showed that glucose and isoleucine were associated with diabetes in PAD patients and glucose (as expected) had the highest discriminant capacity (Figure 2(c)). Hyperlipidemic and normolipemic patients showed significant differences in alanine, aspartate, glucose, isoleucine, lactate, leucine, succinyl-CoA, and valine concentrations and, among them, isoleucine had the highest discriminant capacity (Figure 2(d)). Fumarate, glucose, isoleucine, lactate, malate, serine, and pyruvate were associated with hypertension in 


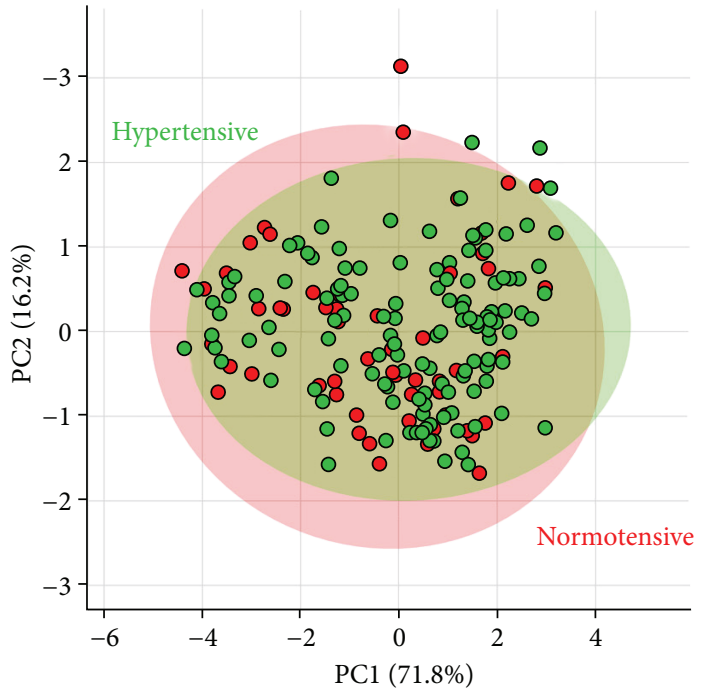

(a)

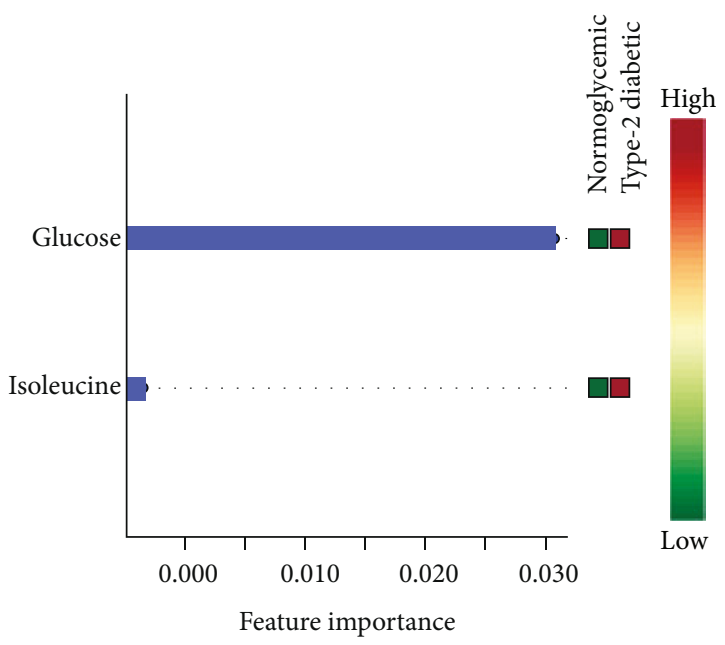

(c)

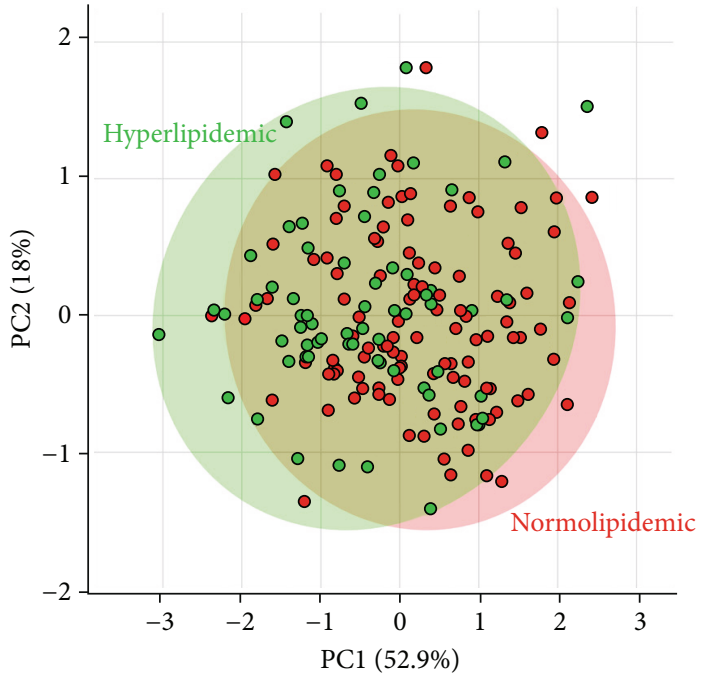

(b)

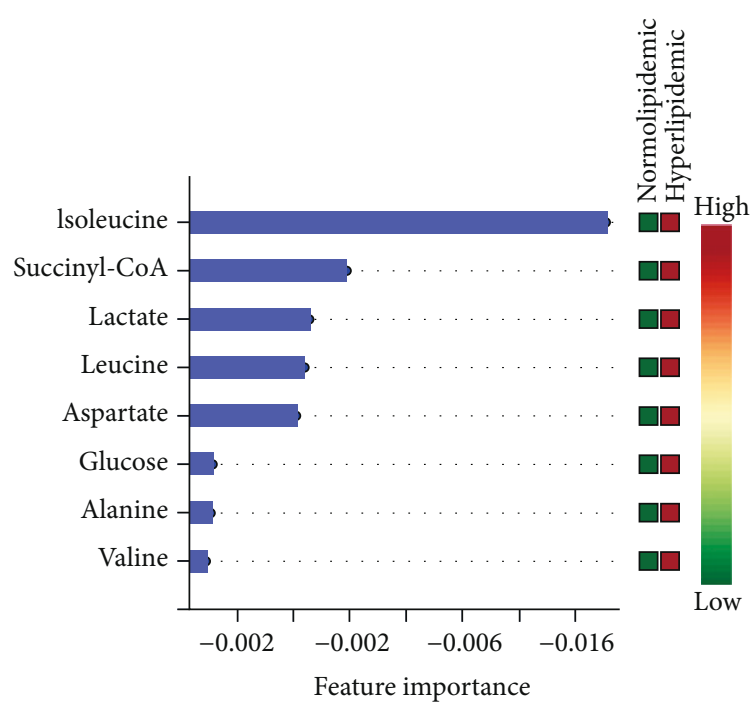

(d)

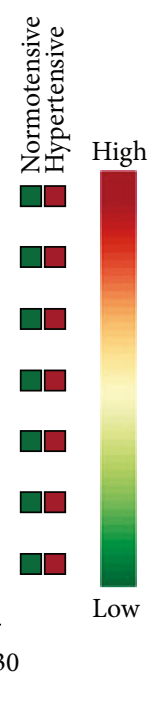

(e)

Figure 2 
TABLE 6: Spearman's correlation coefficients for age, body mass index, and related metabolites.

\begin{tabular}{|c|c|c|c|c|}
\hline & \multicolumn{2}{|c|}{ Age } & \multicolumn{2}{|c|}{ BMI } \\
\hline & Spearman's rho & $p$ value & Spearman's rho & $p$ value \\
\hline 3-Hydroxybutirate & 0.057 & ns & 0.022 & ns \\
\hline Aconitate & 0.205 & 0.003 & -0.236 & 0.010 \\
\hline$\alpha$-Ketoglutarate & 0.054 & ns & -0.031 & ns \\
\hline Alanine & -0.081 & ns & 0.230 & 0.013 \\
\hline Aspartate & 0.083 & ns & 0.256 & 0.005 \\
\hline (Iso)citrate & 0.113 & ns & 0.147 & ns \\
\hline Fumarate & 0.220 & 0.002 & -0.085 & ns \\
\hline Glucose & -0.031 & ns & 0.202 & 0.029 \\
\hline Glutamate & 0.063 & ns & -0.032 & ns \\
\hline Glutamine & 0.007 & ns & 0.157 & ns \\
\hline Isoleucine & 0.124 & ns & 0.194 & 0.036 \\
\hline Lactate & -0.023 & ns & 0.034 & ns \\
\hline Leucine & -0.005 & ns & 0.220 & 0.017 \\
\hline Malate & 0.248 & $<0.001$ & -0.079 & ns \\
\hline Pyruvate & -0.009 & ns & 0.103 & ns \\
\hline Serine & 0.062 & ns & -0.112 & ns \\
\hline Succinate & 0.011 & ns & -0.145 & ns \\
\hline Succinyl-CoA & 0.038 & ns & 0.134 & ns \\
\hline Valine & -0.122 & ns & 0.241 & 0.009 \\
\hline
\end{tabular}

BMI: Body mass index; ns: not significant.

PAD patients, and serine was the metabolite with the best discriminant capacity (Figure 2(e)). Aconitate, fumarate, and malate were associated with age, and aconitate, alanine, aspartate, glucose, isoleucine, leucine, and valine were correlated with BMI (Table 6 and Figure 3). All these metabolites were excluded for further analysis as candidates for PAD biomarker in order to avoid the influence of confounding factors.

3.5. Metabolic Biomarkers of PAD. Metabolites included in further analysis were (iso)citrate, glutamate, succinate, 3hydroxybutirate, $\alpha$-ketoglutarate, and glutamine. From them, (iso)citrate and glutamate were those showing the greatest differences between patients and controls and between the different patient groups according to the Fontaine scale (Figure 4). To evaluate the capacity of these metabolites to discriminate between groups, we performed random forest analyses and ROC curves confirming that (iso)citrate and glutamate were the most powerful metabolites to separate the healthy individuals from PAD patients (Figures 5(a) and 5(b)), and the healthy individuals from asymptomatic or nearly asymptomatic patients (grades I and II), with AUC $>0.95$ (Figures 5(c) and 5(d)). They were also useful to discriminate between the different subgroups of patients (Figure 5(e)).

\section{Discussion}

Energy-balance-associated metabolites are related to oxidative stress and inflammation. We found in PAD patients significant alterations in energy metabolism, particularly evident through the citrate-aconitate-(iso)citrate conversions. The mitochondrial enzymes involved in these reactions are (iso)citrate dehydrogenase (IDH2) and aconitase 2 (ACO2). Both enzymes are crucial for normal mitochondrial function [33]. In mice, decreased IDH2 expression contributes to atherosclerosis progression by increasing oxidative stress [34]. An oxidative environment inactivates aconitase, which in turn undergoes age-dependent oxidative modification. Whether IDH2 and ACO2 may be the cause or consequence of mitochondrial dysfunction in $\mathrm{PAD}$ requires further studies [8].

Mitochondrial dysfunction, oxidative stress, and inflammation are closely related $[24,25]$. The present study shows the existence of significant inverse correlations between various metabolites and PON1-related variables and direct correlations with CCL2. These correlations are stronger for PON1 concentration and lactonase activity. This may be due to the observation that PON1 is located (among other cellular organelles) in the membranes of the mitochondria, protecting them from oxidative stress [25]. Therefore, alterations in the TCA cycle may directly affect PON1. The correlations between metabolites and paraoxonase activity are weaker than those found for lactonase activity, but this is probably due to the differential impact of genetic polymorphisms [23]. Metabolites showing the strongest and more consistent correlations with PON1 and CCl2 were 3-hydroxybutirate, aconitate, (iso)citrate, glutamate, and serine. 3Hydroxybutirate is a ketone body, and studies on the effects of ketones on oxidative stress and inflammation are contradictory. For example, it has been reported that these 

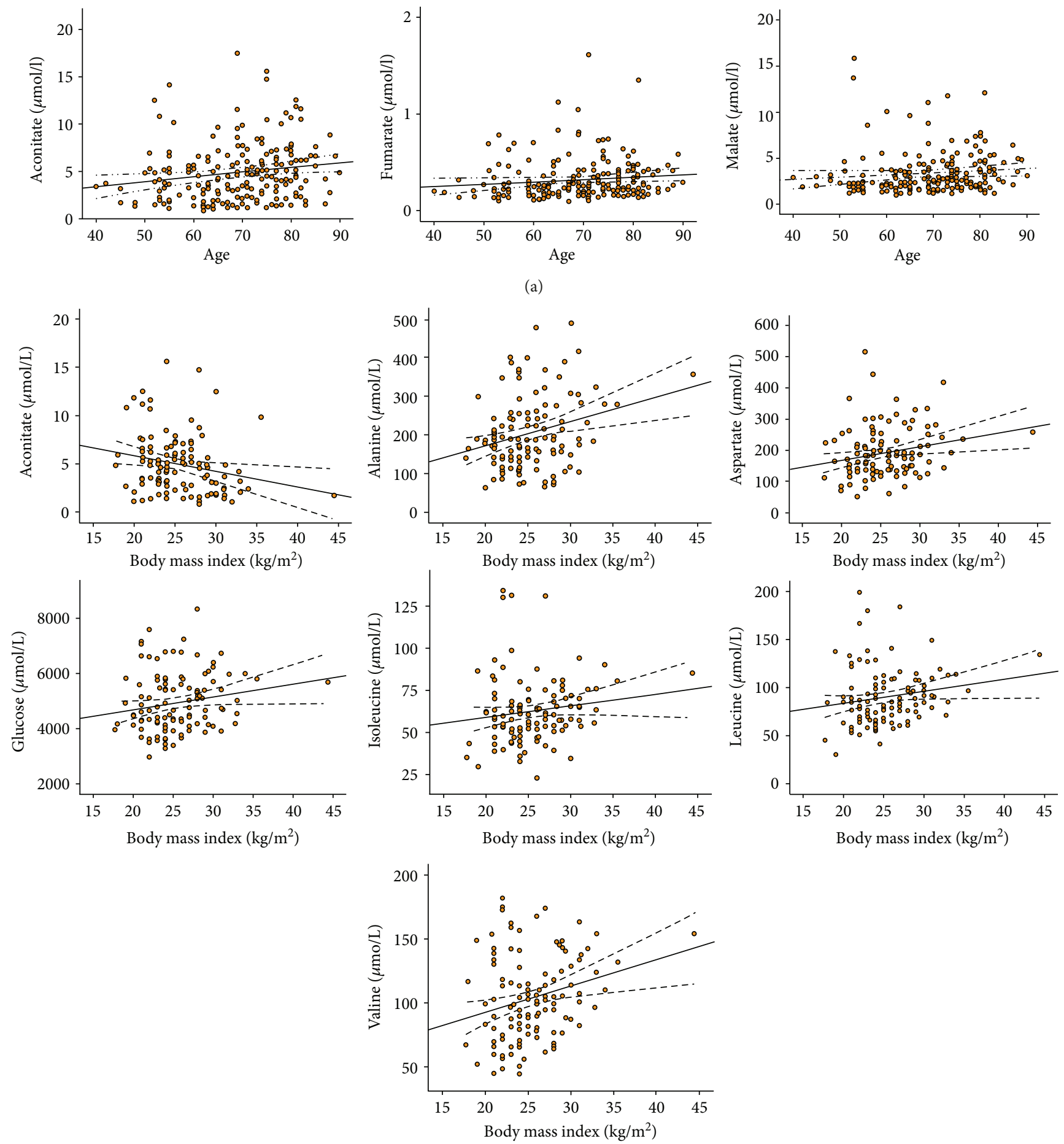

(b)

Figure 3

compounds inhibit mitochondrial production of reactive oxygen species in rat and mice neurons [35-37] while they activate NF- $\kappa \mathrm{B}$, upregulate NADPH oxidase, elevate oxidative stress, and induce the expression of proinflammatory cytokines in endothelial cells and hepatocytes [38, 39]. Our results are in agreement with this latter possibility. It has been suggested that these contradictory findings reflect tissuespecific differences because the source of reactive oxygen species in neurons differ from that in nonneuronal cells [35]. Aconitate is the precursor of itaconate, and this metabolite regulates metabolic remodeling and mitochondrial respiration in inflammatory macrophages [40]. There 


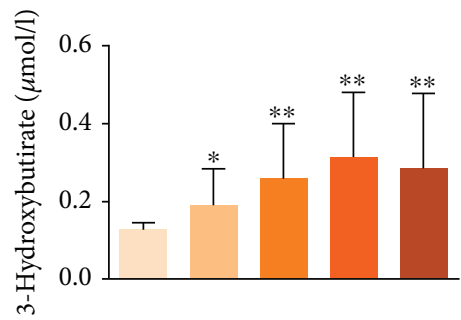

(a)

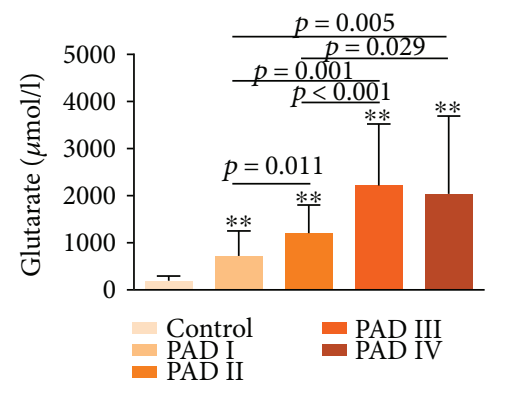

(c)

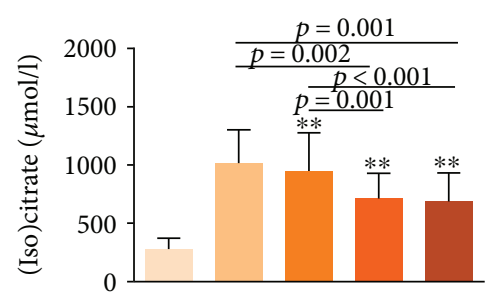

(e)

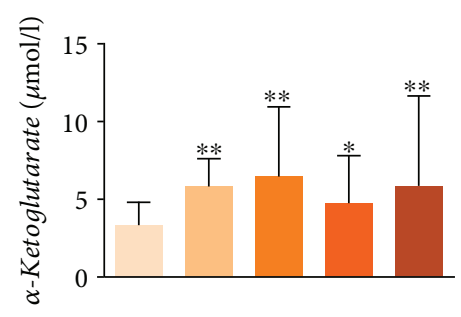

(b)

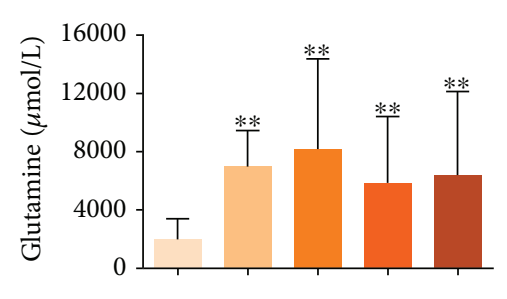

(d)

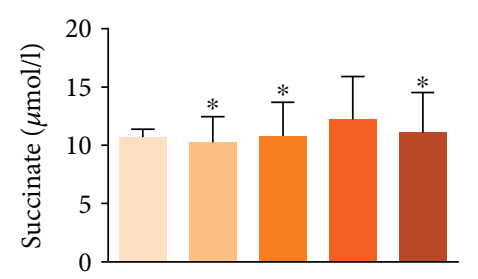

(f)

Figure $4:{ }^{*} p<0.05$ and ${ }^{* *} p<0.001$ for the control samples.

is little information on the associations of (iso)citrate, glutamate, and serine with oxidative stress and inflammation, but studies suggest that glutamate is pro-oxidant and proinflammatory [41] while (iso)citrate and serine may elicit the opposite effect $[42,43]$.

Energy-balance-associated metabolites might be considered as PAD biomarkers. Early diagnosis is important in these patients because preventive treatment has potential benefits in the progression of PAD. Current biomarkers are indeed risk factors [11, 13], and dyslipidemia, hypertension, or diabetes plays independent roles in atherogenesis. In our population, approximately $80 \%$ of patients had one or more of these complications. Their effect on metabolite concentrations is unknown but probably multifactorial. For instance, branched-chain amino acids (BCAA) were influenced by hypertension, diabetes, and dyslipidemia confirming previous associations [44-47]. We therefore discarded metabolites influenced by confounding variables, and we found six candidates with statistically significant differences in concentration between the control group and PAD patients: 3-hydroxybutyrate, $\alpha$-ketoglutarate, glutamate, glutamine, (iso)citrate, and succinate. These candidates were useful to distinguish between PAD patients and the control group and also to discriminate between different clinical stages. The conversion of (iso)citrate to $\alpha$-ketoglutarate is mediated by
IDH2, and increased concentrations of this metabolite have been associated with a worse cardiovascular prognosis [48]. Interestingly, glutamate, another metabolite with a good discriminant capacity, is the substrate for many enzymes located in the mitochondria [49] and plays an important role in the mechanical function of the ischemic myocardium [50]. Understanding glutamate overproduction in the blood of patients with atherosclerosis requires further research. Our more interesting finding indicates that (iso)citrate and glutamate may discriminate healthy participants from PAD patients in the asymptomatic or early symptomatic stages (Fontaine grades I and II). Simple measurements may then provide clinical tools to assess patients at risk but without symptoms. Larger studies using sensitive metabolomic techniques are warranted to confirm these findings and to identify specific metabolic pathways associated with increased risk of PAD.

\section{Conclusion}

Our metabolomic approach supports a relevant association of plasma concentrations of energy-balance-associated metabolites with oxidative stress and inflammation and reveals (iso)citrate and glutamate as candidate biomarkers for discriminating PAD patients without symptomatic disease. 

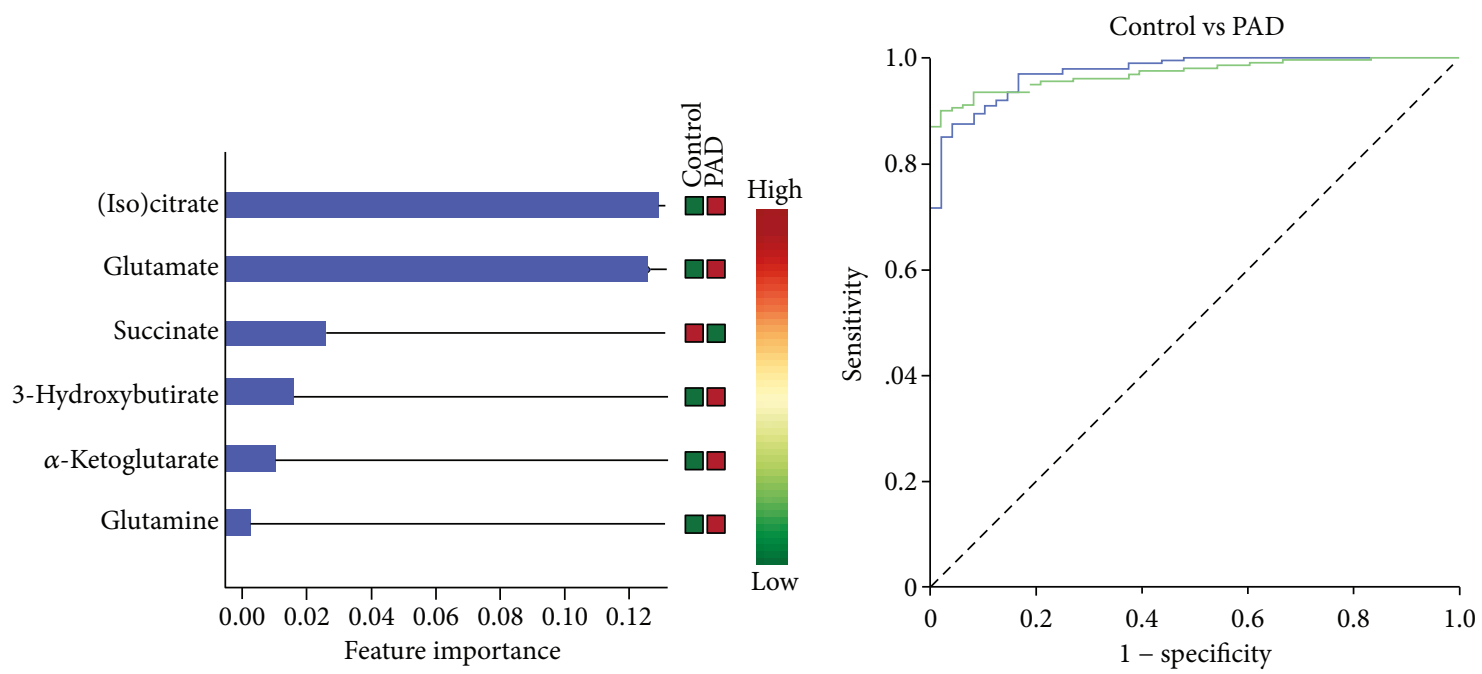

(Iso)citrate AUC: 0.973 (0.955-0.991)

_ Glutamate AUC: 0.970 (0.952-0.988)

(a)

(b)
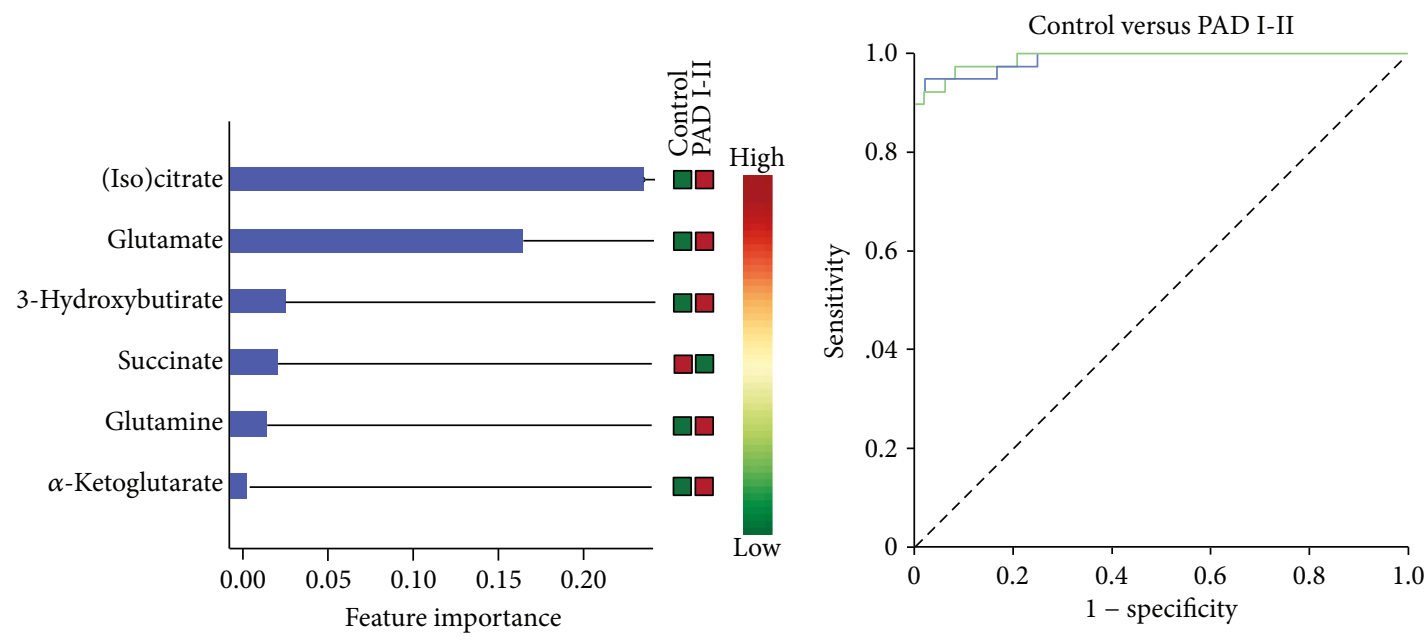

— (Iso)citrate AUC: 0.988 (0.972-1)

— Glutamate AUC: 0.990 (0.977-1)

(c)

(d)

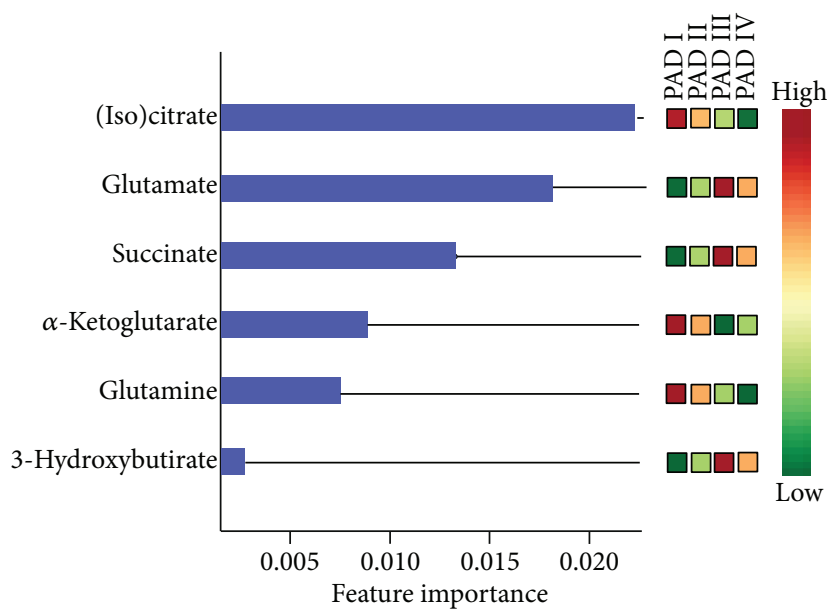

(e)

Figure 5 


\section{Data Availability}

The data used to support the findings of this study are available from the corresponding author upon request.

\section{Conflicts of Interest}

The authors declare that they have no conflicts of interest.

\section{Authors' Contributions}

Anna Hernández-Aguilera and Salvador Fernández-Arroyo contributed equally to this work.

\section{Acknowledgments}

This work was supported by grants from the Plan Nacional de I+D+I, Spain, Instituto de Salud Carlos III (Grant no. PI15/00285, co-founded by the European Regional Development Fund (EU FEDER)) to Jorge Joven and the Ministerio de Ciencia e Innovación (Grant no. SAF2012-38914) to Javier A. Menendez. The authors also acknowledge the support by the Agència de Gestió d'Ajuts Universitaris i de Recerca (AGAUR) (Grant nos. 2014 SGR1227 and 2014 SGR229) and the Fundació La Marató de TV3.

\section{References}

[1] F. G. R. Fowkes, D. Rudan, I. Rudan et al., "Comparison of global estimates of prevalence and risk factors for peripheral artery disease in 2000 and 2010: a systematic review and analysis," Lancet, vol. 382, no. 9901, pp. 1329-1340, 2013.

[2] M. H. Criqui, "Peripheral arterial disease - epidemiological aspects," Vascular Medicine, vol. 6, Supplement 1, pp. 3-7, 2017.

[3] K. E. Wellen and G. S. Hotamisligil, "Inflammation, stress, and diabetes," The Journal of Clinical Investigation, vol. 115, no. 5, pp. 1111-1119, 2005.

[4] G. S. Hotamisligil, "Inflammation and metabolic disorders," Nature, vol. 444, no. 7121, pp. 860-867, 2006.

[5] C. Bernal-Mizrachi, A. C. Gates, S. Weng et al., "Vascular respiratory uncoupling increases blood pressure and atherosclerosis," Nature, vol. 435, no. 7041, pp. 502-506, 2005.

[6] M. Riera-Borrull, E. Rodríguez-Gallego, A. HernándezAguilera et al., "Exploring the process of energy generation in pathophysiology by targeted metabolomics: performance of a simple and quantitative method," Journal of the American Society for Mass Spectrometry, vol. 27, no. 1, pp. 168-177, 2016.

[7] P. Bénit, E. Letouzé, M. Rak et al., "Unsuspected task for an old team: succinate, fumarate and other Krebs cycle acids in metabolic remodeling," Biochimica et Biophysica Acta (BBA) - Bioenergetics, vol. 1837, no. 8, pp. 1330-1337, 2014.

[8] M. Calderón-Santiago, F. Priego-Capote, J. G. Galache-Osuna, and M. D. Luque de Castro, "Method based on GC-MS to study the influence of tricarboxylic acid cycle metabolites on cardiovascular risk factors," Journal of Pharmaceutical and Biomedical Analysis, vol. 74, pp. 178-185, 2013.
[9] K. I. Makris, A. A. Nella, Z. Zhu et al., "Mitochondriopathy of peripheral arterial disease," Vascular, vol. 15, no. 6, pp. 336343, 2007.

[10] G. J. Kemp, "Mitochondrial dysfunction in chronic ischemia and peripheral vascular disease," Mitochondrion, vol. 4, no. 5-6, pp. 629-640, 2004.

[11] S. Hazarika and B. H. Annex, "Biomarkers and genetics in peripheral artery disease," Clinical Chemistry, vol. 63, no. 1, pp. 236-244, 2016.

[12] E. S. H. Kim, K. Wattanakit, and H. L. Gornik, "Using the ankle-brachial index to diagnose peripheral artery disease and assess cardiovascular risk," Cleveland Clinic Journal of Medicine, vol. 79, no. 9, pp. 651-661, 2012.

[13] R. K. Upadhyay, "Emerging risk biomarkers in cardiovascular diseases and disorders," Journal of Lipids, vol. 2015, Article ID 971453, 50 pages, 2015.

[14] J. R. Ussher, S. Elmariah, R. E. Gerszten, and J. R. B. Dyck, "The emerging role of metabolomics in the diagnosis and prognosis of cardiovascular disease," Journal of the American College of Cardiology, vol. 68, no. 25, pp. 2850-2870, 2016.

[15] R. Laaksonen, "Identifying new risk markers and potential targets for coronary artery disease: the value of the lipidome and metabolome," Cardiovascular Drugs and Therapy, vol. 30, no. 1, pp. 19-32, 2016.

[16] G. D. Lewis, A. Asnani, and R. E. Gerszten, "Application of metabolomics to cardiovascular biomarker and pathway discovery," Journal of the American College of Cardiology, vol. 52, no. 2, pp. 117-123, 2008.

[17] J. B. German, B. D. Hammock, and S. M. Watkins, "Metabolomics: building on a century of biochemistry to guide human health," Metabolomics, vol. 1, no. 1, pp. 3-9, 2005.

[18] H. Matthews, J. Hanison, and N. Nirmalan, ““'Omics”informed drug and biomarker discovery: opportunities, challenges and future perspectives," Proteomes, vol. 4, no. 3, 2016.

[19] C. K. Ward-Caviness, T. Xu, T. Aspelund et al., "Improvement of myocardial infarction risk prediction via inflammationassociated metabolite biomarkers," Heart, vol. 103, no. 16, pp. 1278-1285, 2017.

[20] S. E. Ali, M. A. Farag, P. Holvoet, R. S. Hanafi, and M. Z. Gad, "A comparative metabolomics approach reveals early biomarkers for metabolic response to acute myocardial infarction," Scientific Reports, vol. 6, no. 1, article 36359, 2016.

[21] A. Rull, R. Garcia, L. Fernandez-Sender et al., "The role of combined assessment of defense against oxidative stress and inflammation in the evaluation of peripheral arterial disease," Current Molecular Medicine, vol. 11, no. 6, pp. 453-464, 2011.

[22] A. Hernández-Aguilera, J. Sepúlveda, E. Rodríguez-Gallego et al., "Immunohistochemical analysis of paraoxonases and chemokines in arteries of patients with peripheral artery disease," International Journal of Molecular Sciences, vol. 16, no. 12, pp. 11323-11338, 2015.

[23] J. Camps, J. Marsillach, and J. Joven, “The paraoxonases: role in human diseases and methodological difficulties in measurement," Critical Reviews in Clinical Laboratory Sciences, vol. 46, no. 2, pp. 83-106, 2009.

[24] J. Camps, E. Rodríguez-Gallego, A. García-Heredia et al., "Paraoxonases and chemokine (C-C motif) ligand-2 in noncommunicable diseases," Advances in Clinical Chemistry, vol. 63, pp. 247-308, 2014. 
[25] J. Camps, A. García-Heredia, A. Hernández-Aguilera, and J. Joven, "Paraoxonases, mitochondrial dysfunction and noncommunicable diseases," Chemico-Biological Interactions, vol. 259, Part B, pp. 382-387, 2016.

[26] A. Hernández-Aguilera, A. Rull, E. Rodríguez-Gallego et al., "Mitochondrial dysfunction: a basic mechanism in inflammation-related non-communicable diseases and therapeutic opportunities," Mediators of Inflammation, vol. 2013, Article ID 135698, 13 pages, 2013.

[27] S. Novo, "Classification, epidemiology, risk factors, and natural history of peripheral arterial disease," Diabetes, Obesity \& Metabolism, vol. 4, Supplement 2, pp. S1-S6, 2002.

[28] J. Joven, E. Espinel, A. Rull et al., "Serum fatty acid synthase concentration is increased in patients with hepatitis viral infection and may assist in the prediction of liver steatosis," Journal of Clinical Virology, vol. 51, no. 3, pp. 199-201, 2011.

[29] J. Marsillach, G. Aragonès, R. Beltrán et al., “The measurement of the lactonase activity of paraoxonase- 1 in the clinical evaluation of patients with chronic liver impairment," Clinical Biochemistry, vol. 42, no. 1-2, pp. 91-98, 2009.

[30] N. Martinelli, A. García-Heredia, H. Roca et al., "Paraoxonase1 status in patients with hereditary hemochromatosis," Journal of Lipid Research, vol. 54, no. 5, pp. 1484-1492, 2013.

[31] M. Grootveld, "Introduction to the applications of chemometric techniques in 'omics' research: common pitfalls, misconceptions and 'rights and wrongs'," in Metabolic Profiling: Disease and Xenobiotics, M. Grootveld, Ed., Royal Society of Chemistry, Cambridge, 2014.

[32] M. H. Zweig and G. Campbell, "Receiver-operating characteristic (ROC) plots: a fundamental evaluation tool in clinical medicine," Clinical Chemistry, vol. 39, no. 4, pp. 561-577, 1993.

[33] H. J. Ku, Y. Ahn, J. H. Lee, K. M. Park, and J.-W. Park, "IDH2 deficiency promotes mitochondrial dysfunction and cardiac hypertrophy in mice," Free Radical Biology \& Medicine, vol. 80, pp. 84-92, 2015.

[34] X. Fu, X. Huang, P. Li, W. Chen, and M. Xia, "7-Ketocholesterol inhibits isocitrate dehydrogenase 2 expression and impairs endothelial function via microRNA-144," Free Radical Biology \& Medicine, vol. 71, pp. 1-15, 2014.

[35] M. Maalouf, P. G. Sullivan, L. Davis, D. Y. Kim, and J. M. Rho, "Ketones inhibit mitochondrial production of reactive oxygen species production following glutamate excitotoxicity by increasing NADH oxidation," Neuroscience, vol. 145, no. 1, pp. 256-264, 2007.

[36] J. Yin, P. Han, Z. Tang, Q. Liu, and J. Shi, "Sirtuin 3 mediates neuroprotection of ketones against ischemic stroke," Journal of Cerebral Blood Flow and Metabolism, vol. 35, no. 11, pp. 17831789, 2015.

[37] K. Marosi, S. W. Kim, K. Moehl et al., “3-Hydroxybutyrate regulates energy metabolism and induces BDNF expression in cerebral cortical neurons," Journal of Neurochemistry, vol. 139, no. 5, pp. 769-781, 2016.

[38] P. Kanikarla-Marie and S. K. Jain, "Hyperketonemia (acetoacetate) upregulates NADPH oxidase 4 and elevates oxidative stress, ICAM-1, and monocyte adhesivity in endothelial cells," Cellular Physiology and Biochemistry, vol. 35, no. 1, pp. 364-373, 2015.
[39] X. Shi, X. Li, D. Li et al., " $\beta$-Hydroxybutyrate activates the NF- $\kappa \mathrm{B}$ signaling pathway to promote the expression of pro-inflammatory factors in calf hepatocytes," Cellular Physiology and Biochemistry, vol. 33, no. 4, pp. 920-932, 2014.

[40] V. Lampropoulou, A. Sergushichev, M. Bambouskova et al., "Itaconate links inhibition of succinate dehydrogenase with macrophage metabolic remodeling and regulation of inflammation," Cell Metabolism, vol. 24, no. 1, pp. 158-166, 2016.

[41] H. Parfenova, S. Basuroy, S. Bhattacharya et al., "Glutamate induces oxidative stress and apoptosis in cerebral vascular endothelial cells: contributions of HO-1 and HO-2 to cytoprotection," American Journal of Physiology. Cell Physiology, vol. 290, no. 5, pp. C1399-C1410, 2006.

[42] B. A. Paim, J. A. Velho, R. F. Castilho, H. C. F. Oliveira, and A. E. Vercesi, "Oxidative stress in hypercholesterolemic LDL (low-density lipoprotein) receptor knockout mice is associated with low content of mitochondrial NADP-linked substrates and is partially reversed by citrate replacement," Free Radical Biology \& Medicine, vol. 44, no. 3, pp. 444-451, 2008.

[43] M. N. Maralani, A. Movahedian, and Javanmard ShH, "Antioxidant and cytoprotective effects of L-serine on human endothelial cells," Research in Pharmaceutical Sciences, vol. 7, no. 4, pp. 209-215, 2012.

[44] T. J. Wang, M. G. Larson, R. S. Vasan et al., "Metabolite profiles and the risk of developing diabetes," Nature Medicine, vol. 17, no. 4, pp. 448-453, 2011.

[45] J. E. Ho, M. G. Larson, A. Ghorbani et al., "Metabolomic profiles of body mass index in the Framingham heart study reveal distinct cardiometabolic phenotypes," PLoS One, vol. 11, no. 2, article e0148361, 2016.

[46] P. Yang, W. Hu, Z. Fu et al., "The positive association of branched-chain amino acids and metabolic dyslipidemia in Chinese Han population," Lipids in Health and Disease, vol. 15, no. 1, p. 120, 2016.

[47] T. Fujii, S. Yura, K. Tatsumi et al., "Branched-chain amino acid supplemented diet during maternal food restriction prevents developmental hypertension in adult rat offspring," Journal of Developmental Origins of Health and Disease, vol. 2, no. 3, pp. 176-183, 2011.

[48] S. Cheng, M. G. Larson, E. L. McCabe et al., "Distinct metabolomic signatures are associated with longevity in humans," Nature Communications, vol. 6, no. 1, p. 6791, 2015.

[49] N. C. Danbolt, “Glutamate uptake," Progress in Neurobiology, vol. 65, no. 1, pp. 1-105, 2001.

[50] L. M. Dinkelborg, R. K. Kinne, and M. K. Grieshaber, “Transport and metabolism of L-glutamate during oxygenation, anoxia, and reoxygenation of rat cardiac myocytes," The American Journal of Physiology, vol. 270, 5, Part 2, pp. H1825-H1832, 1996. 


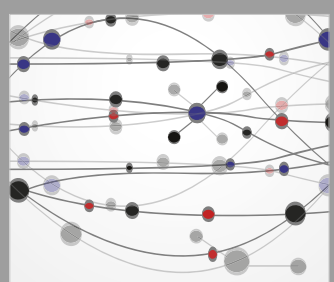

The Scientific World Journal
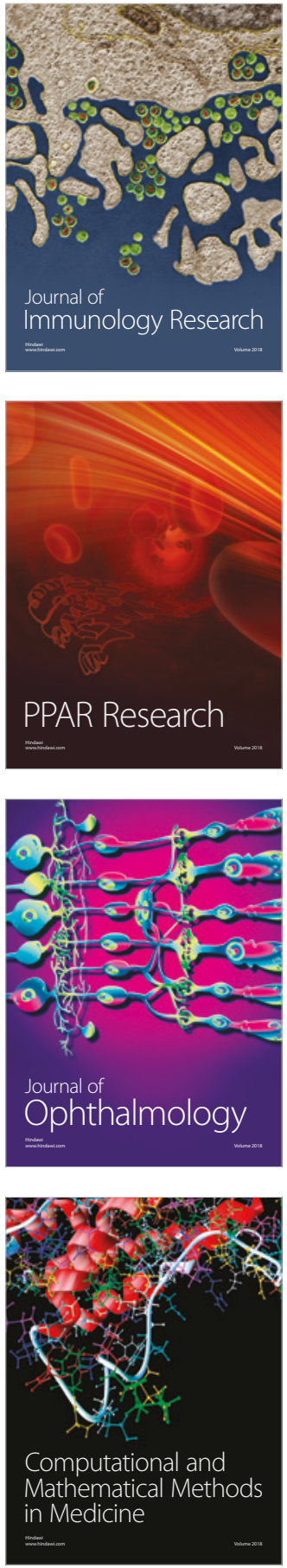

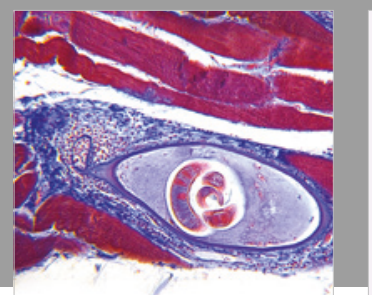

Gastroenterology Research and Practice

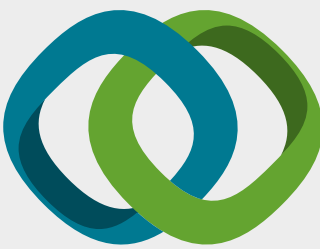

\section{Hindawi}

Submit your manuscripts at

www.hindawi.com
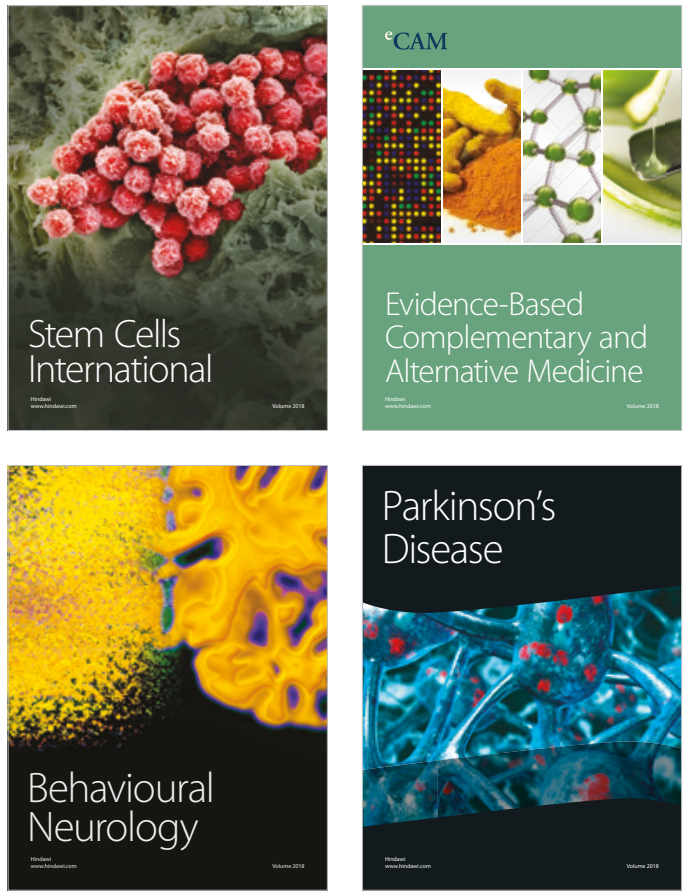

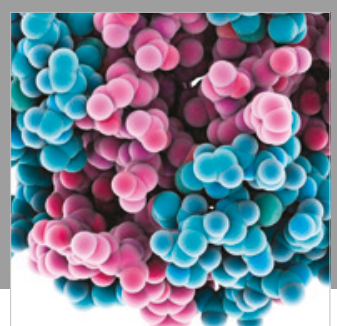

ournal of

Diabetes Research

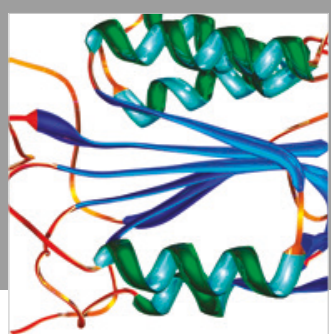

Disease Markers
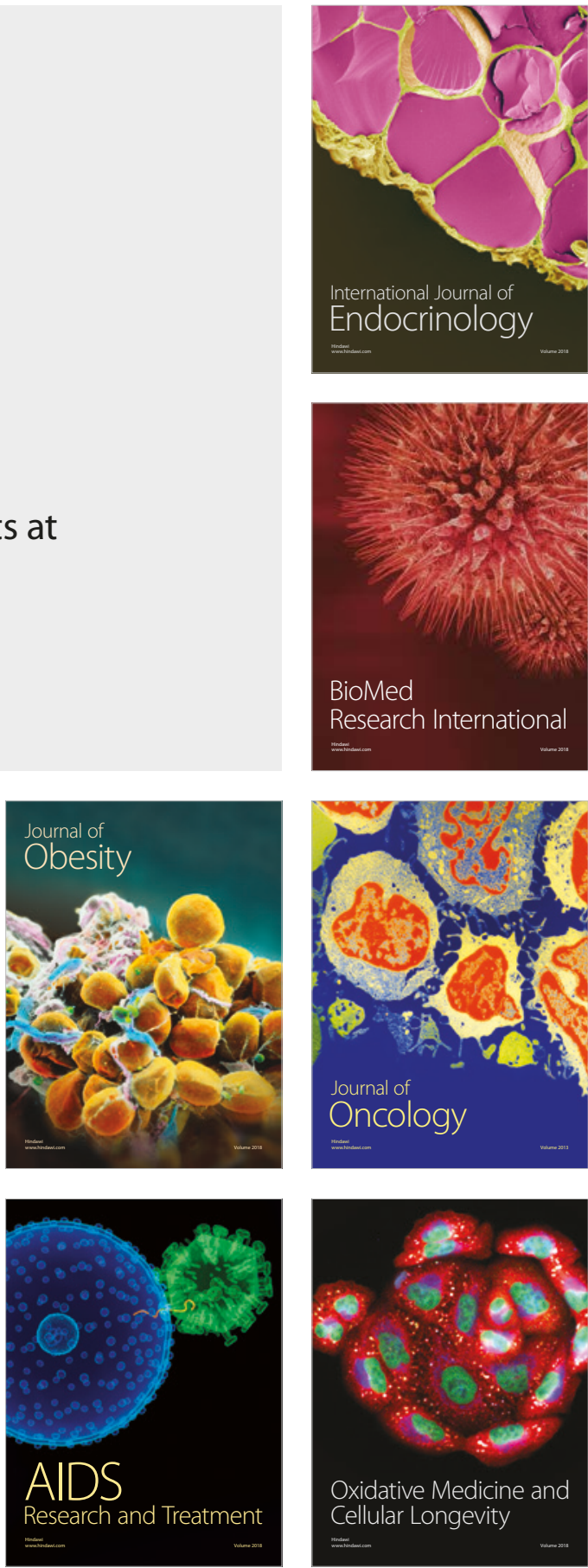